\title{
OTOLOGY
}

\section{Cross-cultural adaptation and Italian validation of chronic otitis media outcome test 15 (COMOT-15)}

\author{
Adattamento culturale e validazione in italiano del questionario COMOT-15 \\ nella valutazione degli outcomes del trattamento dell'otite media cronica
}

\author{
Michele Cavaliere ${ }^{1}$, Pasquale Capriglione ${ }^{1}$, Flavia Cavaliere², Eugenio De Corso ${ }^{3}$, Elisabetta Zanoletti ${ }^{4}$, Gaetano Motta ${ }^{5}$, \\ Maurizio lengo ${ }^{1}$, Elena Cantone ${ }^{1}$ \\ ${ }^{1}$ Department of Neuroscience, Reproductive and Odontostomatological Sciences - ENT section, University "Federico II", Naples, \\ Italy; ${ }^{2}$ Department of Humanities, Philology Section, University of Naples Federico II, Naples, Italy; ${ }^{3}$ Policlinico Universitario A. \\ Gemelli IRCCS, UOC Otorinolaringoiatria. Università Cattolica del Sacro Cuore, Rome, Italy; ${ }^{4}$ Department of Neuroscience DNS, \\ Otolaryngology Section, Padova University, Padova, Italy; ${ }^{5}$ Otorhinolaryngology, Head and Neck Surgery Unit, Department of Mental \\ and Physical Health and Preventive Medicine, Università degli Studi della Campania Luigi Vanvitelli, Naples, Italy
}

\section{SUMMARY}

Objective. Chronic suppurative otitis media (CSOM) may result in a serious impairment of the quality of life (QoL). The most relevant characteristics of CSOM are well represented in the "Chronic Otitis Media Outcome Test 15" questionnaire (COMOT-15). The aim of the study was to translate and adapt the COMOT-15 questionnaire into Italian.

Methods. This is a prospective study conducted on 52 Caucasian Italian-speaking patients with CSOM and 52 controls. The linguistic adaptation of the COMOT-15 consisted of the translation of the English version into Italian and reliability assessment of the translation. Psychometric measurements included internal consistency and test-retest reliability.

Results. Cronbach's $\alpha$ was 0.95 . The test-retest reliability showed a strong positive correlation for total score and all subscales. Age and gender had no influence on the scores. The correlation between the COMOT-15 and the pure tone average showed a significant association for the scale "Hearing Function".

Conclusions. The Italian COMOT-15 questionnaire provides good internal consistency and is suitable for QoL evaluation in Italian-speaking patients suffering from CSOM. In addition, it is able to evaluate the subjective symptoms perceived by patients.

KEY WORDS: QoL, chronic otitis, COMOT 15, hearing loss, ear discharge

\section{RIASSUNTO}

Obiettivo. L'otite media cronica suppurativa (OMCS) può determinare una grave compromissione della qualità della vita $(Q o L)$ dei pazienti. Gli aspetti clinici più rilevanti dell'OMCS sono rappresentati nel questionario "Chronic Otitis Media Outcome Test 15" (COMOT-15). Scopo dello studio è tradurre e adattare alla lingua italiana il questionario COMOT-15.

Metodi. Abbiamo effettuato uno studio prospettico di validazione arruolando 52 pazienti con diagnosi di OMCS e 52 controlli. Il processo di adattamento linguistico del questionario è consistito nella traduzione della versione originale inglese in italiano e nella misura dell'affidabilità della stessa. Le misure psicometriche che abbiamo preso in considerazione includevano coerenza interna e affidabilità test-retest.

Risultati. Il Cronbach $\alpha$ era pari a 0,95. Abbiamo riscontrato una forte correlazione positiva sia per il punteggio totale che per tutte le sottoscale. Età e sesso non hanno influenzato i punteggi. L'analisi delle correlazioni tra COMOT-15 e soglia uditiva per la via aerea ha mostrato un'associazione significativa per la sottoscala "Funzione uditiva".

Conclusioni. Sulla base dei nostri risultati è stato possibile dimostrare che la versione italiana del questionario COMOT-15 da noi proposta ha una buona coerenza interna ed è particolarmente adatta per la valutazione della QoL nei pazienti di lingua italiana affetti da otite media cronica suppurativa. Inoltre, esso è in grado di valutare i sintomi soggettivi percepiti dai pazienti.

PAROLE CHIAVE: QoL, otite cronica, COMOT-15, ipoacusia, otorrea
Received: October 16, 2020

Accepted: December 3, 2020

\section{Correspondence}

Eugenio De Corso

Fondazione Policlinico Universitario "A. Gemelli - IRCCS, Catholic University of the Sacred Heart largo A. Gemelli 8, 00168 Rome, Italy

Tel. +3906 30154439; Fax +3906 3051194

E-mail: eugenio.decorso@policlinicogemelli.it

Funding

None.

Conflict of interest

The Authors declare no conflict of interest.

How to cite this article: Cavaliere M, Capriglione $\mathrm{P}$, Cavaliere F, et al. Cross-cultural adaptation and Italian validation of chronic otitis media outcome test 15 (COMOT-15). Acta Otorhinolaryngol Ital 2021;41:277-281. https://doi. org/10.14639/0392-100X-N1184

(C) Società Italiana di Otorinolaringoiatria e Chirurgia Cervico-Facciale

\section{(c) (1) $(9)$}

This is an open access article distributed in accordance with the CC-BY-NC-ND (Creative Commons Attribution-NonCommercial-NoDerivatives 4.0 International) license. The article can be used by giving appropriate credit and mentioning the license, but only for non-commercial purposes and only in the original version. For further information: https:// creativecommons.org/licenses/by-nc-nd/4.0/deed.en 


\section{Introduction}

Otitis media (OM) includes various subtypes of pathologies characterised by middle ear mucosa inflammation such as acute otitis media (AOM), otitis media with effusion (OME), chronic suppurative otitis media (CSOM), mastoiditis and cholesteatoma. CSOM (with or without cholesteatoma) affects approximately $2-4 \%$ of the general population and is defined as a chronic inflammation of the middle ear and mastoid cavity with or without tympanic membrane perforation ${ }^{1-3}$. It is characterised by recurrent ear discharge and hearing loss, and, less frequently, otalgia, fever, vertigo and tinnitus ${ }^{1}$.

CSOM may be associated with significant limitation of hearing function that may result in communication problems impairing social interaction and professional life. Conductive hearing loss is considered a serious disability, contributing to a significant disease burden worldwide ${ }^{4,5}$. In addition, persistent discharge from the ear, pain or frequent physician visits may result in a serious impairment of patients' quality of life $(\mathrm{QoL})^{4,5}$. In case of CSOM with cholesteatoma, complications such as facial nerve paralysis, Bezold's, Luc's, Citelli's abscesses, meningitis or encephalitis, may develop and potentially threaten the patient's life ${ }^{6}$.

QoL measures are clinically important to determine the impact of the disease, as well as the effectiveness of different types of treatment, and assessment of objective parameters does not mirror the subjective experiences of patients. Health-related QoL is particularly important in chronic health conditions, such as CSOM, requiring longterm treatment and life-long care. Generally, hearing levels measured by pure tone audiometry have been used to define and quantify CSOM-related QoL. However, disabling hearing loss on audiometric data may not correlate with QoL ${ }^{7}$. Assessing subjectively QoL with a specific questionnaire is steadily increasing in clinical medicine and, in particular, in different otorhinolaryngological diseases as rhinosinusitis, several studies have reported that physicians' ratings of the QoL in patients with chronic illness were significantly different from patients' self-rated QoL ${ }^{5-7}$. Currently, in clinical practice, there are a large number of questionnaires that can measure QoL ${ }^{5-7}$. However, not all questionnaires are able to evaluate the subjective symptoms, functional outcomes and the psychological aspects of CSOM ${ }^{4-6}$.

Generic questionnaires, as the Short Form 36 Health Survey (SF-36), can be used to assess CSOM-related discomfort, but they are not disease-specific and can, therefore, underestimate the impact of the disease on patients' QoL. Hence, disease-specific measures are more sensitive than generic measures ${ }^{5}$. So far, the most relevant characteristics of CSOM, including clinical symptoms and functional and psychological impairments, are well represented in the COMOT-15 questionnaire ${ }^{6}$. However, it is crucial to develop questionnaires in native languages and ensure they are appropriate to the culture of the country so that they can be used on larger groups of the population. Thus, we developed an Italian COMOT-15 questionnaire, which subjectively assesses disease-specific QoL.

The aim of this study was to linguistically and culturally adapt the COMOT-15 questionnaire into Italian and to obtain measures of its internal consistency and test-retest reliability.

\section{Materials and methods}

\section{Study population and study design}

This is a prospective instrument validation study that was conducted on 52 Caucasian Italian-speaking patients affected by CSOM who were diagnosed and followed at our institution. Patients were enrolled between January 2018-December 2018 at ENT section of Department of Neuroscience, Reproductive and Odontostomatological Sciences, University "Federico II" of Naples, Italy. We enrolled as controls 52 Caucasian Italian-speaking healthy volunteers. A diagnosis of CSOM was obtained from the patient's medical history, ENT examinations, including otomicroscopy and oto-endoscopy, pure tone audiometry and computed tomography (CT) scans. All subjects were asked to fill in the translated COMOT-15 questionnaire on their own during their first visit to the outpatient clinic and again one month after their first visit for retest evaluation.

The linguistic adaptation process of the Italian version of the COMOT-15 questionnaire consisted of two phases: the first phase was translation of the original English version into Italian, and the second consisted of measuring the reliability of the translation obtained.

Pure-tone audiometry for testing conventional frequency range $(0.125$ to $8 \mathrm{kHz})$, was performed using an Amplaid 319 audiometer (Amplaid Inc., Milan, Italy) in a double-walled, soundproof room. Pure-tone average values were calculated in $\mathrm{dB}$ from the air conduction hearing loss as the mean of $0.5,1,2$ and $4 \mathrm{kHz}$ thresholds (pure tone average, PTA).

Subjects signed an informed consent form before participating in the study. All procedures performed in studies were in accordance with the ethical standards of the institutional research committee and with the 1964 Helsinki declaration and its later amendments or comparable ethical standards. Local Board of Medical Ethics number 339/20.

\section{COMOT-15 questionnaire}

The COMOT-15 consists of 3 subscales, categorised as ear symptoms (questions 1-6), hearing function (questions 7-9) 
and mental health (questions 10-13), which form the overall score. In addition to questions from 1 to 13 , the COMOT-15 contains two other questions: a general evaluation of the impact of CSOM on QoL (question 14) and a question on the frequency of ENT visits as a result of CSOM in the previous 6 months (question 15). The total score and the 3 subscales were transformed into a 0-100 score by dividing the sum of the raw scores of the items by the sum of spans of the items, followed by multiplying this number by $100^{4}$.

\section{Linguistic adaptation process of the Italian version of the COMOT-15}

The linguistic adaptation was obtained by translation of the original version in English into Italian and back-translation into English again. Two native Italian otolaryngologists and one bilingual expert whose mother tongue was Italian independently translated the questionnaire. The 3 versions were unified by consensus between the translator and researchers. The bilingual translator back-translated this final version. After the back-translation, the two otolaryngologists and the bilingual expert adapted the Italian questionnaire in order to improve the semantic equivalence of meaning between the two English versions. After these steps had been completed, the first version of the Italian COMOT-15 questionnaire was conceived. This version was initially pilot-tested on a sample of 25 healthy volunteers to determine whether the questions could be clearly understood. The pilot test was performed by a single investigator (EC) through a direct interview to assess possible difficulties in understanding the questionnaire. After some words were modified to facilitate comprehension, the Italian translation was finalised (Fig. 1).

\section{Measuring the reliability of the cross adapted Italian version of COMOT-15}

In a second phase of this study, we compared patients diagnosed with CSOM with healthy volunteers. Exclusion criteria were as follows: age $<18$, lack of full legal capacity, inability to read and understand Italian, immunodeficiency, diabetes, heart, oncological, immunological, malformations, neuropsychiatric diseases and previous ear surgery.

\section{Data analysis}

The data were analysed with SPSS 19 for Windows (IBM Corp., Armonk, NY). Continuous baseline characteristics were presented as the mean and standard deviation. The internal consistency of the test was determined using Cronbach's $\alpha$. The test-retest reliability was assessed by exploring the correlation between the COMOT-15 scores obtained during the first visit at the outpatient clinic (test) and those obtained one month after the first visit (retest) by using the Pearson correlation coefficient.

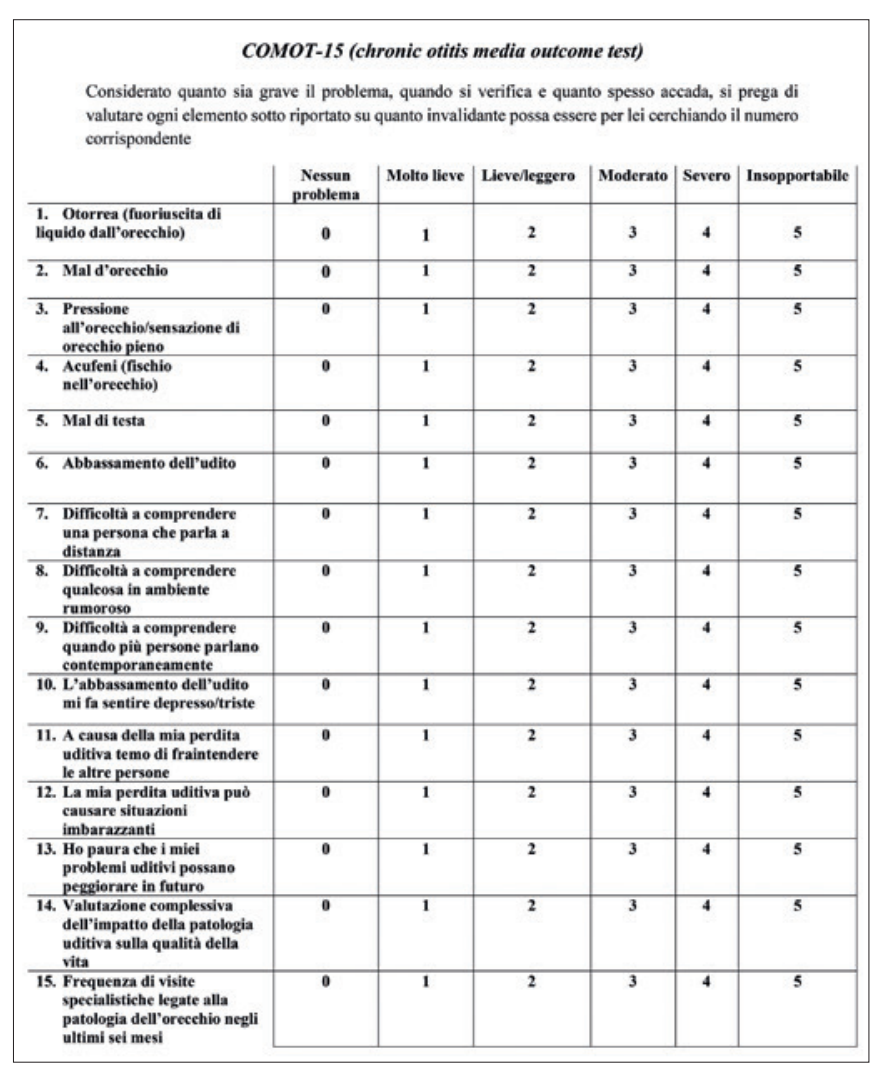

Figure 1. The Italian version of COMOT-15 questionnaire.

The absolute scores of the healthy participants and patients were analysed with the non-parametric MannWhitney U-test to assess the validity of the questionnaire. Statistical significance was achieved for a $p$ inferior to 0.05 .

\section{Results}

We recruited 52 Caucasian Italian-speaking patients (mean age was $48.3 \pm 16.1 \mathrm{SD}$, range $18-64$ years; 28 females) diagnosed with CSOM and 52 Caucasian Italian-speaking healthy volunteers (mean age 42.6 $\pm 10.1 \mathrm{SD}$, range 19-62; 29 females). The male/female ratio was 0.8 in the investigational arm and 0.7 in the control arm.

We found a bilateral CSOM in $13 / 52$ subjects (25\% of cases) and 38/52 (73\%) CSOM with cholesteatoma. Pure tone average (PTA) $[\mathrm{dB}]$ calculated from air conduction hearing loss at $0.5,1,2$ and $4 \mathrm{kHz}$ in CSOM patients showed a value of $55[\mathrm{~dB}] \pm 10.2 \mathrm{SD}$. The opposite ear showed a healthy aspect in 39/52 $(75 \%)$ patients.

The total score of the Italian version of COMOT-15 was $36.76 \pm 13.1($ mean $\pm \mathrm{SD})$. The mean scores of the subscales of COMOT-15 were $11.05 \pm 4.45($ mean \pm SD) for ear symptoms, $13.48 \pm 5.83$ (mean $\pm \mathrm{SD}$ ) for hearing function and $9.69 \pm 4.65$ (mean \pm SD) for mental health 


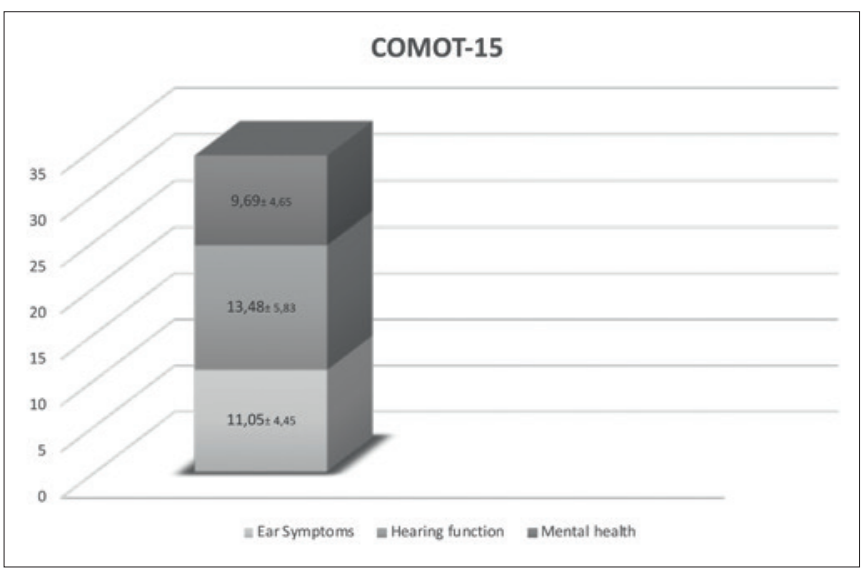

Figure 2. Mean scores of the Italian version of COMOT-15. The mean total score of the Italian version of COMOT-15 was $36.76 \pm 3.1$. The mean scores of the subscales of COMOT-15 were $11.05 \pm 4.45$ for ear symptoms, $13.48 \pm 5.83$ for hearing function, and $9.69 \pm 4.65$ for mental health (mean \pm SD).

(Fig. 2). The mean scores of subgroups, CSOM with/without cholesteatoma, are reported in Table I.

The Mann-Whitney U-test showed that the COMOT-15 scores were significantly higher in patients with CSOM than in control subjects $(\mathrm{U}=68.5, \mathrm{p}=0.0001)$.

The internal consistency was supported by a Cronbach's $\alpha$ of 0.95 . The test-retest reliability showed a strong, positive Pearson correlation coefficient $(r=0.98, p=0.00001)$ for total score and all subscales: ear symptoms $\mathrm{p}<0.01$, hearing function $\mathrm{p}<0.01$ mental health $\mathrm{p}<0.01$ (Fig. 3). Age and gender had no influence on the evaluation of the scores of COMOT-15 ( $\mathrm{p}>0.05)$.

The analysis of correlations between the scales of the COMOT-15 and the pure tone average showed a significant association for the scale "Hearing Function", p $<0.008$.

\section{Discussion}

The present study aimed to translate the COMOT-15 into the Italian language. The translated version was administered to a large study population.

The results showed that the Italian COMOT-15 provides good reliability as well as internal consistency, suggesting its suitability in determining the QoL of Italian-speaking individuals suffering from CSOM. The mean total score of

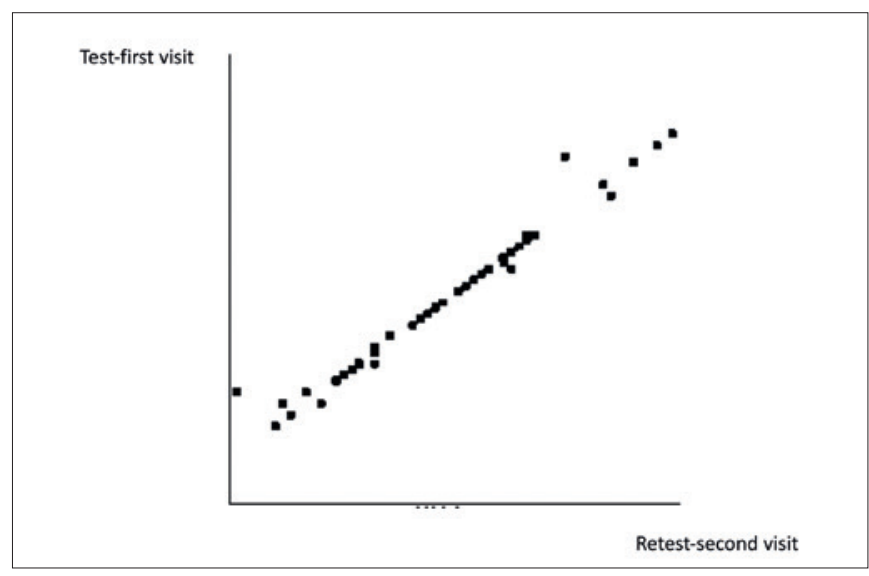

Figure 3. Test-retest reliability. The test-retest reliability showed a strong, positive Pearson correlation coefficient $(r=0.98, p=0.00001)$ for total score and all subscales: ear symptoms $p<0.01$, hearing function $p<0.01$, mental health $p<0.01$.

the Italian COMOT-15 (36.76 \pm 13.1 , mean \pm SD) suggested a deterioration of QoL in patients affected by CSOM. In addition, the mean scores of the subscales pointed out that ear symptoms were the major cause of reduced QoL in Italian-speaking individuals with CSOM.

QoL measurements in CSOM subjects assess the impact of the disease on patient's overall health, social interaction and psychological status ${ }^{6}$. The most common CSOM QoL questionnaires, although each with some limitations, are: the Glasgow Benefit Inventory (GBI), Glasgow Health Status Inventory (GHSI), Chronic Ear Survey (CES) ${ }^{8}$, Chronic Otitis Media Questionnaire 12 (COMQ-12), Otitis Media-6 questionnaire (OM-6) and the health-related QoL (HRQoL) ${ }^{5,6,9-11}$. The GBI was designed to evaluate changes in QoL following surgery, whereas the GHSI measures the impact of a health problem on the QoL and provides information about the physical health subscale. Although the GBI and GHSI have been commonly used for ear, nose and throat diseases, they do not give enough information about the hearing function and mental health of the patient ${ }^{5}$.

The CES questionnaire includes some single items evaluating the frequency of symptoms or medical problems, but it does not include a subjective assessment of their severity as perceived by the patients ${ }^{4,7,8}$. It does not seem to adequately cover the subjective point of view of the patients ${ }^{4,8}$.

Table I. Mean scores of the Italian version of COMOT-15 in subgroups (CSOM with cholesteatoma and without cholesteatoma). Differences between total cases scores and subgroups (CSOM with/without cholesteatoma) scores are not statistically significant (mean \pm SD).

\begin{tabular}{lccccc} 
& No. & Overall score & Ear symptoms & Hearing function & Mental health \\
Total cases & 52 & $36.76 \pm 13.1$ & $11.05 \pm 4.45$ & $13.48 \pm 5.83$ & $9.69 \pm 4.65$ \\
CSOM with cholesteatoma & 38 & $37.02 \pm 13.43$ & $11.41 \pm 4.19$ & $13.92 \pm 5.49$ & $10.01 \pm 4.38$ \\
CSOM without cholesteatoma & 14 & $36.07 \pm 12.76$ & $10.08 \pm 4.64$ & $12.29 \pm 6.07$ & $8.84 \pm 4.84$ \\
\hline
\end{tabular}


The COMQ-12 consists of 12 questions grouped into four categories on severity of symptoms, impact on lifestyle, work and the health service, as well as general impact on the patient's QoL. However, like the GBI and GHSI it does not give information about hearing function and mental health of the patient ${ }^{1,4,6}$.

Lastly, the OM-6 and the HR QoL are intended exclusively for paediatric use ${ }^{12,13}$.

However, the most relevant characteristics of CSOM ${ }^{14,15}$, including clinical symptoms and functional and psychological impairments, are well represented in the COMOT-15 ${ }^{4,6}$. The COMOT-15 seems to represent a well-conceived synthesis of the most important items affecting patients' quality of life, representing the impact of the disease and of its therapy.

Indeed, the 15 questions of COMOT- 15 consist of three subscales, categorised as ear symptoms, hearing function and mental health. In addition, the COMOT-15 contains a question on the general evaluation of the impact of CSOM on QoL and a question about the frequency of ENT visits ${ }^{4,6}$.

The COMOT-15 is currently the only questionnaire that evaluates the frequency of ENT visits, which represents an indirect measure of QoL ${ }^{4,6}$. For these reasons, it seems to be the most suitable questionnaire to evaluate CSOM. Furthermore, the COMOT-15 questionnaire assesses the impact of CSOM on the patient's overall health, social interaction and psychological status ${ }^{6}$.

Given the usefulness of QoL questionnaires, in our opinion it is essential to develop QoL measures in native languages and appropriate to the culture of the country. There has been growing literature exploring tools that are able to assess clinical patient-based outcomes, or rather, outcomes as experienced by the patients. In addition, a subjective way of investigation, like a questionnaire, can reflect QoL much more closely than exclusive clinician-rated outcomes. Furthermore, it is crucial to develop native-languages questionnaires that can be used on larger groups of patients ${ }^{7}$.

\section{Conclusions}

This study confirmed the importance of using validated translations of QoL questionnaires, which, by giving the possibility to compare populations from different countries and with different cultures, represent a valid tool to establish clinical protocols for global health systems. Our study showed that the Italian COMOT-15 questionnaire has good internal consistency and validity, and can be successfully applied to Italian-speaking patients. Given that the COMOT-15 is a quick, easy-to-use instrument to determine the QoL of subjects with CSOM, it is strongly advisable that further translations in other different languages will follow.

\section{References}

1 Nadol JB Jr, Staecker H, Gliklich RE. Outcomes assessment for chronic otitis media: the Chronic Ear Survey. Laryngoscope 2009;110:3235. https://doi.org/10.1097/00005537-200003002-00009

2 Cavaliere M, Di Lullo AM, Cantone E, et al. Cholesteatoma vs granulation tissue: a differential diagnosis by DWI-MRI apparent diffusion coefficient. Eur Arch Otorhinolaryngol 2018;275:2237-2243. https:// doi.org/10.1007/s00405-018-5082

3 Cavaliere M, Di Lullo AM, Caruso A, et al. Diffusion-weighted intensity magnetic resonance in the preoperative diagnosis of cholesteatoma. ORL 2014;76:212-221. https://doi.org/10.1159/000365931

4 Baumann I, Gerendas B, Plinkert PK, et al. General and disease-specific quality of life in patients with chronic suppurative otitis media: a prospective study. Health Qual Life Outcomes 2011;9:48. https://doi. org/10.1186/1477-7525-9-48.

5 Maile EJ, Youngs R. Quality of life measures in otitis media. J Laryngol Otol 2013;127:442-447. https://doi.org/10.1017/ S0022215113000509

6 Prabhu P, Chandrashekar A, Jose A, et al. Development and administration of chronic suppurative otitis media questionnaire-12 (COMQ-12) and chronic otitis media outcome test-15 (COMOT-15) in Kannada. Int Arch Otorhinolaryngol 2018;22:108-112. https://doi. org/10.1055/s-0037-1603644

7 Cantone E, Castagna G, Sicignano S, et al. Impact of intranasal sodium hyaluronate on the short-term quality of life of patients undergoing functional endoscopic sinus surgery for chronic rhinosinusitis. Int Forum Allergy Rhinol 2014;4:484-487. https://doi.org/10.1002/alr.21310

8 Lucidi D, De Corso E, Paludetti G, et al. Quality of life and functional results in canal wall down vs canal wall up mastoidectomy. Acta Otorhinolaryngol Ital 2019;39:53-60. https://doi. org/10.14639/0392-100X-2005

9 Redfors YD, Jönsson R, Tideholm B, et al . Psychometric properties of the swedish version of the Glasgow Benefit Inventory in otosclerosis subjects. Laryngoscope Investig Otolaryngol 2019;4:673-677. https://doi.org/10.1002/lio2.320

10 Hawthorne G, Hogan A. Measuring disability-specific patient benefit in cochlear implant programs: developing a short form of the Glasgow Health Status Inventory, the Hearing Participation Scale. Int J Audiol 2002;41:535-544. https://doi.org/10.3109/14992020209056074

11 Ralli G, Milella C, Ralli M, et al. Quality of life measurements for patients with chronic suppurative otitis media: Italian adaptation of "Chronic Ear Survey". Acta Otorhinolaryngol Ital 2017;37:51-57. https://doi.org/10.14639/0392-100X-1041

12 Indius JH, Alqaderi SK, Kjeldsen AD, et al. Middle ear disease in Danish toddlers attending nursery day-care - Applicability of OM-6, disease specific quality of life and predictors for middle ear symptoms. Int J Pediatr Otorhinolaryngol 2018;110:130-134. https://doi. org/10.1016/j.ijporl.2018.04.031

13 Grindler DJ, Blank SJ, Schulz KA, et al. Impact of otitis media severity on children's quality of life. Otolaryngol Head Neck Surg 2014;151:333-340. https://doi.org/10.1177/0194599814525576

14 Pareschi R, Lepera D, Nucci R. Canal wall down approach for tympano-mastoid cholesteatoma: long-term results and prognostic factors. Acta Otorhinolaryngol Ital 2019;39:122-129. https://doi. org/10.14639/0392-100X-2237

15 De Corso E, Cantone E, Galli J, et al. Otitis media in children: Which phenotypes are most linked to allergy? A systematic review. Pediatr Allergy Immunol 2021;32:524-534. https://doi.org/10.1111/ pai.13431. 